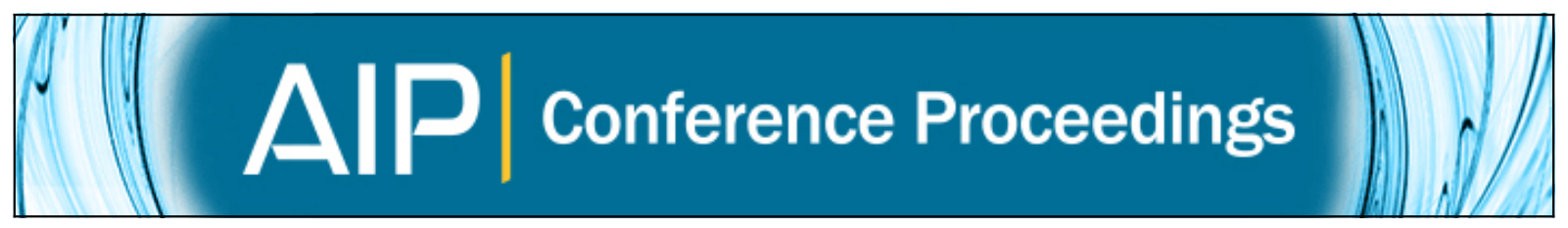

\title{
Observations of a 3 He -rich SEP Event over a Broad Range of Heliographic Longitudes: Results from STEREO and ACE
}

M. E. Wiedenbeck, G. M. Mason, R. Gómez-Herrero, D. Haggerty, N. V. Nitta, C. M. S. Cohen, E. E. Chollet, A.

C. Cummings, R. A. Leske, R. A. Mewaldt, E. C. Stone, T. T. von Rosenvinge, R. Müller-Mellin, M. Desai, and

U. Mall

Citation: AIP Conference Proceedings 1216, 621 (2010); doi: 10.1063/1.3395943

View online: http://dx.doi.org/10.1063/1.3395943

View Table of Contents: http://scitation.aip.org/content/aip/proceeding/aipcp/1216?ver=pdfcov

Published by the AIP Publishing

\section{Articles you may be interested in}

3 He -rich SEP events observed by STEREO-A

AIP Conf. Proc. 1539, 139 (2013); 10.1063/1.4811007

Observations of the 3D coronal structure over a solar cycle

AIP Conf. Proc. 1216, 691 (2010); 10.1063/1.3395962

Modeling CMEs in three dimensions using an analytic MHD model

AIP Conf. Proc. 471, 645 (1999); 10.1063/1.58817

Quantitative results on heating events in the quiet corona

AIP Conf. Proc. 471, 67 (1999); 10.1063/1.58785

Quiet solar wind signatures above active regions observed in X-rays

AIP Conf. Proc. 471, 231 (1999); 10.1063/1.58752 


\title{
Observations of a ${ }^{3} \mathrm{He}$-rich SEP Event over a Broad Range of Heliographic Longitudes: Results from STEREO and ACE
}

\author{
M. E. Wiedenbeck* , G. M. Mason ${ }^{\dagger}$, R. Gómez-Herrero** , D. Haggerty ${ }^{\dagger}$, \\ N. V. Nitta ${ }^{\ddagger}$, C. M. S. Cohen ${ }^{\S}$, E. E. Chollet ${ }^{\S}$, A. C. Cummings ${ }^{\S}$, R. A. Leske ${ }^{\S}$, \\ R. A. Mewaldt ${ }^{\S}$, E. C. Stone ${ }^{\S}$, T. T. von Rosenvinge`, R. Müller-Mellin**, M. Desai ${ }^{*}$ \\ and U. Mall ${ }^{\dagger \dagger}$ \\ * Jet Propulsion Laboratory, California Institute of Technology, Pasadena, CA 91109 USA \\ ${ }^{\dagger}$ Johns Hopkins University/Applied Physics Laboratory, Laurel, MD 20723 USA \\ ${ }^{* *}$ Universität Kiel, 24118 Kiel, Germany \\ ${ }^{*}$ Lockheed Martin Solar and Astrophysics Lab, Palo Alto, CA 94304 USA \\ ${ }^{\S}$ California Institute of Technology, Pasadena, CA 91125 USA \\ INASA/Goddard Space Flight Center, Greenbelt, MD 20771 USA \\ " Southwest Research Institute, San Antonio, TX 78238 USA \\ ${ }^{\dagger}$ Max-Planck-Institut für Sonnensystemforschung, Lindau, Germany
}

\begin{abstract}
Observations of energetic ions and electrons from STEREO and ACE have been used to investigate the longitudinal extent of particle emissions from ${ }^{3} \mathrm{He}$-rich solar energetic particle (SEP) events. In the event of 3-4 Nov 2008, ions and electrons were detected $20^{\circ}$ ahead and behind the nominal connection from the source region to $1 \mathrm{AU}$, and electrons were also detected $60^{\circ}$ ahead. The results are consistent with those of earlier studies that correlated data from near-Earth spacecraft with Helios data or with observations of source regions on the Sun.
\end{abstract}

Keywords: solar energetic particles, solar flares, ${ }^{3} \mathrm{He}$-rich SEP events, particle transport PACS: $96.50 . \mathrm{Vg}, 96.60 . \mathrm{Vg}$

\section{INTRODUCTION}

In the prevailing model of ${ }^{3} \mathrm{He}$-rich solar energetic particle (SEP) events, acceleration of ions and electrons is powered by magnetic reconnection at the site of a solar flare [1]. When the reconnection involves some open magnetic field lines, energetic particles can escape into the heliosphere. It is expected that the longitudinal distribution of these escaping particles should be relatively narrow due to the localized nature of the source region. This expectation is supported by studies comparing single-point observations of ${ }^{3} \mathrm{He}$-rich SEP events at $1 \mathrm{AU}$ with the locations of the associated flare sites on the Sun $[1,2]$, as well as by a study combining $\mathrm{He}$ isotope data from Helios-1 \& -2 and ISEE-3 [3]. However, another investigation using Helios and near-Earth data [4] showed that electrons from impulsive SEP events frequently have much broader longitudinal distributions. One of the goals of the NASA STEREO mission is to make multipoint in situ measurements of SEP events while imaging their source regions in order to better understand acceleration and transport processes in these events.

From the time of STEREO commissioning in January 2007 to May 2009, the two STEREO spacecraft separated from each other by $>90^{\circ}$ in heliolongitude. This period was characterized by very low solar activity (there were no sunspots on more than $1 / 2$ of the days) and few ${ }^{3} \mathrm{He}$-rich SEP events were detected by spacecraft at $1 \mathrm{AU}$ [5]. However, because of the quiet Sun and the low event rate, associations between observations of the same event at multiple spacecraft and with activity imaged on the Sun could be made with little risk of confusion.

We report observations of ${ }^{3} \mathrm{He}$-rich SEP events made using the LET, SEPT, and SIT instruments on STEREO$A$ and $-B$ and the SIS, EPAM, and ULEIS instruments on ACE. Imaging data from the SECCHI instruments on STEREO and the LASCO and EUVI instruments on SOHO as well as radio burst data from the Waves instruments on both STEREO and Wind were used to associate the in situ observations with activity on the Sun. A preliminary version of this work was presented in [6].

\section{${ }^{3}$ HE-RICH SEP EVENTS}

Events were selected based on the observation of a measurable increase of ${ }^{3} \mathrm{He}$ intensity $\left(\gtrsim 10^{-4} / \mathrm{cm}^{2} \mathrm{srs} \mathrm{MeV} / \mathrm{nuc}\right)$ in the energy range $2.3-$ 3.8 MeV/nuc in the LET instrument on STEREO-A and/or -B. The other data sources mentioned above were 
then checked for additional detections of these events or of their solar sources. The two STEREO spacecraft carry identical instrument payloads and thus the species and energy coverage as well as the sensitivity are essentially the same. The ACE instrument designs differ from those on STEREO and these differences need to be taken into account in comparing observations.

The 24 January 2007 Event: The largest of the ${ }^{3} \mathrm{He}-$ rich events detected from 2007 through mid-2009 occurred on 24 January 2007 when the two STEREO spacecraft were near Earth, essentially collocated (longitudinal separation $\sim 0.5^{\circ}$ ) with one another and with ACE. Although large in comparison with the other event discussed in this paper, the 24 January 2007 event was rather small in comparison to many of the ${ }^{3} \mathrm{He}$-rich SEP events observed closer to solar maximum. For example, the ${ }^{3} \mathrm{He}$ fluence at $5 \mathrm{MeV} /$ nuc in the 24 January 2007 event was a factor $\sim 20$ less than in the extensively studied event of 9 September 1998 [7], which was far from the largest ${ }^{3} \mathrm{He}-$ rich event of solar cycle 23 .

This 24 January event originated from active region 10939 located at $\sim \mathrm{S} 04 \mathrm{~W} 60$, which produced a number of B-class X-ray flares. Thus the observing spacecraft were well-connected to the flare site by a nominal Parkerspiral magnetic field. Type III radio bursts, indicating the escape of low-energy electrons (typically $<20 \mathrm{keV}$ ) from the Sun, were observed in conjunction with these events, and electrons with energies extending to above $100 \mathrm{keV}$ were detected by the spacecraft at 1 AU. Figure 1 compares the energy spectra of ${ }^{3} \mathrm{He}$ and ${ }^{4} \mathrm{He}$ measured by the STEREO/LET instruments and by the combination of ULEIS and SIS on ACE. There is good agreement between the spectra measured using the LETs and SIS in the 5-10 MeV/nuc energy interval where their measurements overlap. Extrapolation of the STEREO energy spectra to below $1 \mathrm{MeV} /$ nuc also yields reasonable agreement with measurements from ULEIS. The ${ }^{3} \mathrm{He} /{ }^{4} \mathrm{He}$ ratio, measured to be $\sim 1$ above $\sim 2 \mathrm{MeV} /$ nuc, decreases towards lower energies and reaches a value of $\sim 0.1$ at $0.3 \mathrm{MeV} /$ nuc.

From February 2007 through October 2008 (21 months), no clear increase in the ${ }^{3} \mathrm{He}$ intensity that would indicate a ${ }^{3} \mathrm{He}$-rich event was detected by either of the STEREO/LET instruments. However, CIR events were common during this period and at times it would have been possible for a small, low-energy ${ }^{3} \mathrm{He}$-rich event to be missed due to spillover from CIR ${ }^{4} \mathrm{He}$.

The 3-4 November 2008 Event: This event occurred when the STEREO spacecraft had each separated from Earth by $41^{\circ}$ in heliolongitude. The particles detected at 1 AU were associated with solar active region 11007, located at N35W38 as viewed from Earth. Solar observations [5] showed a C1 x-ray flare, type III radio bursts, and a small CME. The source region was nearly radially aligned with STEREO-A. The Parker spiral magnetic

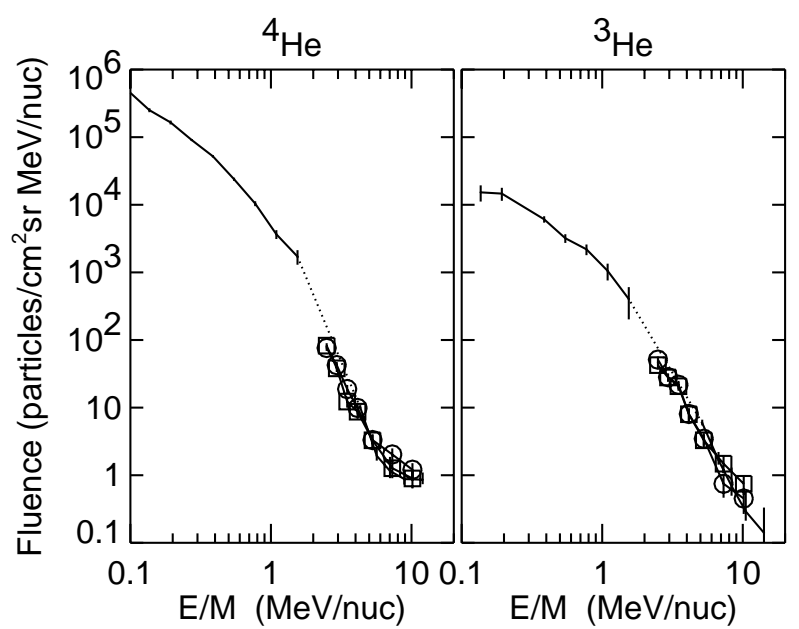

FIGURE 1. Energy spectra of ${ }^{4} \mathrm{He}$ (left panel) and ${ }^{3} \mathrm{He}$ (right panel) measured in the ${ }^{3} \mathrm{He}$-rich SEP event of 24 January 2007 , when ACE and the two STEREO spacecraft were all located near Earth. Solid lines show spectra measured on ACE using ULEIS below $2 \mathrm{MeV} /$ nuc and SIS at higher energies. Dotted lines connect the highest energy ULEIS point with the lowest energy SIS point. Open circles (open squares) show measurements from LET on STEREO-A (STEREO-B). At high energies the ${ }^{3} \mathrm{He} /{ }^{4} \mathrm{He}$ ratio is close to unity, but below $1 \mathrm{MeV} /$ nuc ${ }^{3} \mathrm{He}$ has a harder spectrum than ${ }^{4} \mathrm{He}$, resulting in a decrease of the ${ }^{3} \mathrm{He} /{ }^{4} \mathrm{He}$ ratio with decreasing energy. The flattening of the ${ }^{4} \mathrm{He}$ spectrum near $10 \mathrm{MeV} /$ nuc is due to anomalous cosmic rays (ACRs).

field calculated assuming a $400 \mathrm{~km} / \mathrm{s}$ solar wind would reach 1 AU approximately midway between STEREO$\mathrm{B}$ and ACE. This connection is illustrated in the upper panel of Figure 2 where the dotted line shows the nominal field line and the dark grey region shows a band extending $\pm 20^{\circ}$ about the nominal connection. This width corresponds approximately to the $\pm 1 \sigma$ spread of heliolongitudes over which ${ }^{3} \mathrm{He}$-rich events have been observed relative to their associated $\mathrm{x}$-ray flare events $[1,2]$. The light shaded region extends to $\pm 2 \sigma$. Mason et al. [5] report that the potential field source surface (PFSS) model corresponding to the time of the 3-4 November 2008 event does not indicate the presence of open field lines connecting the active region to the ecliptic plane, in apparent contradiction to the fact that the observations reported here show that particles had access to a wide range of heliographic longitudes in the ecliptic.

The intensity versus time plots in Figure 2 show observations of electrons, He isotopes, and $\mathrm{O}$ and $\mathrm{Fe}$ ions from the three spacecraft. The energy ranges used, which have been chosen to match as closely as possible between STEREO and ACE, are listed in the figure caption. The observations of ${ }^{4} \mathrm{He}$ at STEREO-A and ACE were dominated by a CIR event at this time and have been omitted. Although the ion intensities are low, the SEP event 


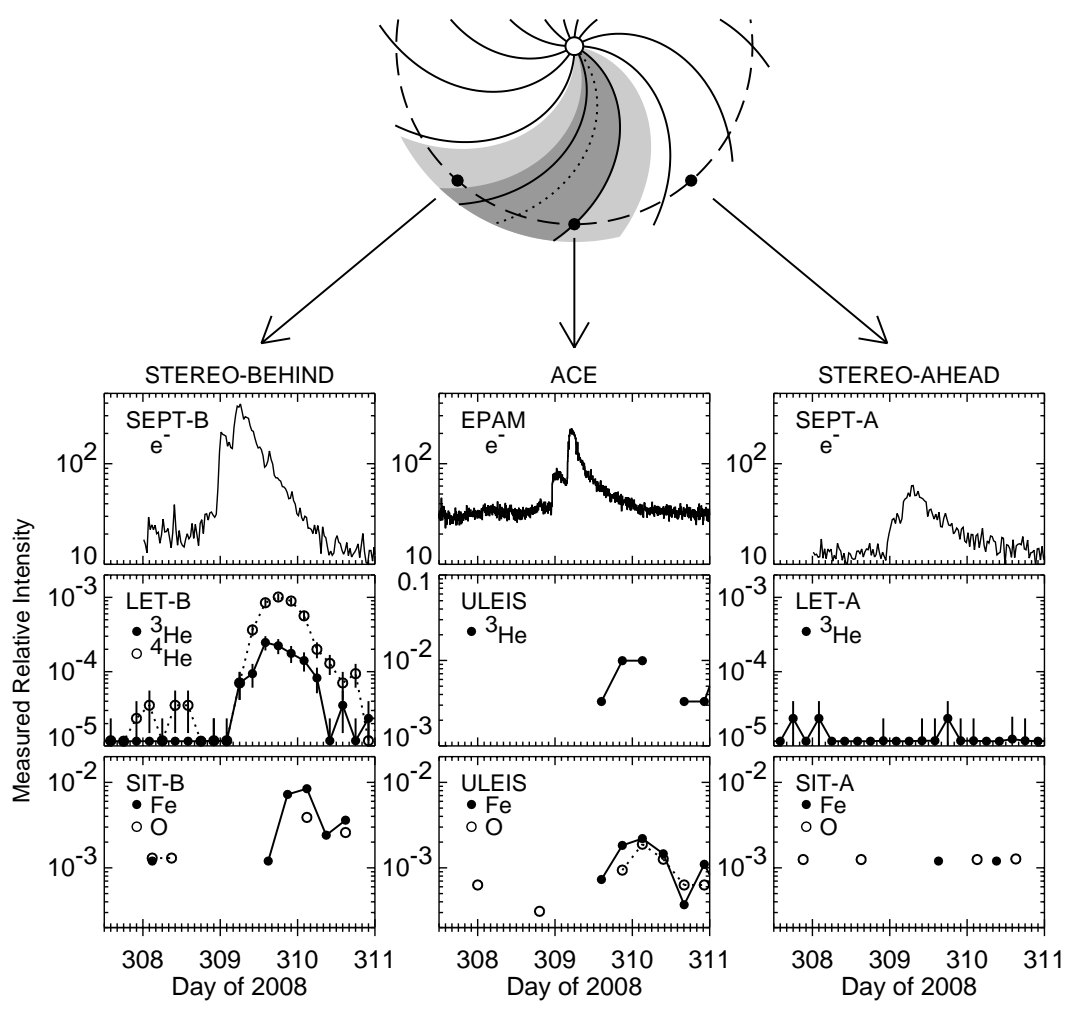

FIGURE 2. Observations of solar energetic particles from the event(s) of 3-4 November 2008 (day of year 308-309). Left column: STEREO-Behind; center column, ACE; right column, STEREO-Ahead. Upper row shows electrons from STEREO/SEPT $(65-105 \mathrm{keV})$ and ACE/EPAM $(62-103 \mathrm{keV})$. Middle row shows ${ }^{3} \mathrm{He}$ (filled circles and solid lines) and ${ }^{4} \mathrm{He}$ (open circles and dotted lines) from STEREO/LET (2.3-3.8 MeV/nuc) and ACE/ULEIS (0.32-0.45 MeV/nuc). Bottom row shows Fe (filled circles and solid lines) and $\mathrm{O}$ (open circles and dotted lines) from STEREO/SIT and ACE/ULEIS (0.32-0.45 MeV/nuc). The drawing indicates the locations of the three spacecraft in their 1 AU orbit (dashed line). The nominal Parker spiral field line (for $V_{\mathrm{SW}}=400 \mathrm{~km} / \mathrm{s}$ ) from the flare site is shown as the dotted line. The dark and light shaded bands indicate spreads of $\pm 20^{\circ}$ and $\pm 40^{\circ}$, respectively, about the nominal field line.

is clearly seen from the increases in ${ }^{3} \mathrm{He}$ and in the $\mathrm{Fe} / \mathrm{O}$ ratio at STEREO-B and ACE. At STEREO-A, however, the ${ }^{3} \mathrm{He}, \mathrm{O}$, and $\mathrm{Fe}$ intensities are near background levels with no indication of the SEP event. The observation of ion increases at locations $\sim 20^{\circ}$ ahead and behind the nominal connection to the active region is consistent with the previously-reported heliolongitude distribution [1,2], as is the absence of a detection at STEREO-A with its $\sim 60^{\circ}$ separation from the nominal field line.

The electron data from STEREO-B and ACE show evidence for at least two distinct injections. At STEREO-A the electron event(s) are also observed, but with a distinctly slower rise to maximum and lower peak intensity than at STEREO-A. With the slower rise, multiple injections are not as clearly discernible.

\section{DISCUSSION}

In the 3-4 November 2008 event, energetic electrons were detected at all three spacecraft (Fig. 2), in spite of the large $\left(82^{\circ}\right)$ spread in heliolongitude. On the other hand, ions were detected only at STEREO-B and ACE, both within $\sim 20^{\circ}$ from the nominal connection to the flare site, and not at STEREO-A, which had a connection angle of $\sim 60^{\circ}$. These results are consistent with the few previous multipoint studies of ${ }^{3} \mathrm{He}$-rich events. Wibberenz \& Cane [4] investigated electron events using data from Helios- $1 \&-2$ and IMP-8 and found cases in which electrons were detected by spacecraft separated by $80^{\circ}$ or more in longitude. Reames and collaborators [3] used the two Helios spacecraft plus ISEE-3 to search for events in which ${ }^{3} \mathrm{He}$ could be detected at two or three spacecraft, requiring similar ${ }^{3} \mathrm{He} /{ }^{4} \mathrm{He}$ ratios at the different spacecraft to confirm the association. They found only three events during 1979-1982 that met their criteria, and they concluded that it is typically possible to detect ions from ${ }^{3} \mathrm{He}$-rich events only within $\sim 20^{\circ}$ of the field line from the flare. (They did report one example [10 February 1979] in which an event may have been detected with three spacecraft spread over $\sim 60^{\circ}$ in longitude and with one of the spacecraft possibly as far as $90^{\circ}$ east of the 
flare site, but it was not possible to determine whether all three spacecraft observed consistent ${ }^{3} \mathrm{He} /{ }^{4} \mathrm{He}$ ratios in this event.) Reames et al. [3] concluded that the ion data are consistent with rather narrow longitudinal distributions for the ions. A direct comparison between electron and ion longitudinal distributions in the Helios events has not been reported; the lists of 3-spacecraft electron events from [4] and of 2- or 3-spacecraft ${ }^{3} \mathrm{He}$ events from [3] have no events in common.

Particles in the 3-4 Nov 2008 event extended over wide ranges of heliolongitude: ions over $\geq 40^{\circ}$ and electrons over $\geq 80^{\circ}$. It is not clear whether the apparently different longitudinal extents of the ion and electron distributions is a characteristic feature of ${ }^{3} \mathrm{He}$-rich events, as one might conclude from comparing the electron results of Wibberenz \& Cane [4] with the ion measurements of Reames et al. [3], or if it might instead be due to instrument sensitivity limitations. A quantitatively useful comparison of intensity-time profiles between electrons and ions is difficult because differences in particle speeds and rigidities can result in significant differences in interplanetary diffusion coefficients and adiabatic energy losses. However, we note that the peak electron intensity at STEREO-A is a factor $\sim 7$ less than that at STEREO-B (see Fig. 2), and if the ${ }^{3} \mathrm{He}$ intensities at the two STEREOs differed by this same factor, the ${ }^{3} \mathrm{He}$ count rate in the STEREO-A LET would have been only marginally detectable (a few counts per day) in this event.

If one assumes that the lack of ion detections at STEREO-A in the 3-4 November 2008 event is actually due to the ions having a significantly narrower longitudinal distribution than the electrons, one can speculate about the possible mechanism responsible for this effect. There are several reported differences between SEP electrons and SEP ions from flare-associated events that could be related to such an effect. Observations of electron spectra extending smoothly down to energies of a few $\mathrm{keV}$ [8] in some events have been used to argue that electron acceleration must take place relatively high in the corona, since the overlying column density of matter in the low corona would prevent the escape of such low energy electrons. On the other hand, the increase in the ionic charge state of $\mathrm{Fe}$ at energies around $0.5 \mathrm{MeV} / \mathrm{nuc}$ [9] is thought to be due to stripping by Coulomb collisions. The relatively high density required to achieve the necessary stripping on the time scale of a flare event suggests a source in the low corona.

Another difference is found between the locations where hard X-rays and gamma-rays are emitted from a flare event on the Sun $[10,11]$. In some events, the $x$-ray sources, which are produced by electrons interacting at footpoints of magnetic loops, are not collocated with the gamma-ray sources produced by interacting ions, indicating that the acceleration regions for electrons and ions are not identical. Thus it is possible that the two populations could be escaping into the heliosphere on different open field lines, which could lead to different longitudinal distributions. If the spread of longitudes for electrons is systematically broader than that for ions, as suggested by the Helios studies [3, 4] and supported by our work (assuming our non-detection of ions at STEREO-A is not due to sensitivity limitations), these differences may provide significant constraints on the geometry involved in the reconnection and acceleration processes.

These measurements also bear on the suggestion of Wibberenz \& Cane [4] that the wide longitudinal extents of electron events could be the result of propagation in the low corona carrying the particles to open field lines remote from the flare site. Because of their higher velocities, electrons may be able to reach such field lines more readily than ions in the time that is available.

Multipoint measurements in additional ${ }^{3} \mathrm{He}$-rich SEP events will be needed to determine 1) how far away in longitude from their solar sources electrons and ions from ${ }^{3} \mathrm{He}$-rich events can be observed and 2) whether the longitudinal distributions of electrons and ions actually differ significantly from one another. With the increase in solar activity expected in 2009 or 2010 , the two STEREO spacecraft, in combination with ACE, are well positioned to search for events with particle detections far from the nominal connection to the flare site and to test the adequacy of present ideas about the escape of flareaccelerated particles from the Sun and their transport in the inner heliosphere.

\section{ACKNOWLEDGMENTS}

This work was supported by NASA at Caltech (under grant NNX08AI11G and through UC Berkeley under contract NAS5-03131), JPL, GSFC, and APL.

\section{REFERENCES}

1. D. V. Reames, Space Sci. Rev., 90, 413, 1999.

2. N. V. Nitta et al., ApJ, 650, 438, 2006.

3. D. V. Reames, M.-B. Kallenrode, \& R. G. Stone, ApJ, 380, 287, 1991.

4. G. Wibberenz \& H. V. Cane, ApJ, 650, 1199, 2006.

5. G. M. Mason et al., ApJL, 700, L56, 2009.

6. M. E. Wiedenbeck et al., Proc. 31st Internat. Cosmic Ray Conf. (Łódź), paper \#1279, 2009.

7. G. M. Mason et al., ApJ, 574, 1039, 2002.

8. D. W. Potter, R. P. Lin, \& K. A. Anderson, ApJL, 236, L97, 1980.

9. B. Klecker, E. Möbius, \& M. A. Popecki, Space Sci. Rev., 130, 273, 2007.

10. R. P. Lin et al., ApJL, 595, L69, 2003.

11. G. J. Hurford et al., ApJL, 644, L93, 2006. 